\title{
Ostrowski type inequalities for functions whose derivatives are strongly $(\alpha, m)$-convex via $k$-Riemann-Liouville fractional integrals
}

\author{
Seth Kermausuor
}

\begin{abstract}
In this paper, we provide some Ostrowski type integral inequalities for functions whose derivatives in absolute value at some powers are strongly $(\alpha, m)$ convex with modulus $\mu \geq 0$ via the $k$-Riemann-Liouville fractional integrals. Similar results related to $(\alpha, m)$-convex functions are obtained as a particular case.
\end{abstract}

Mathematics Subject Classification (2010): 26A33, 26A51, 26D10, 26D15.

Keywords: Ostrowski's inequality, strongly convex functions, strongly $(\alpha, m)$ convex functions, Riemann-Liouville fractional integrals, Hölder's inequality.

\section{Introduction}

Recall that given an interval $I$ in $\mathbb{R}$, a function $f: I \rightarrow \mathbb{R}$ is said to be convex if

$$
f(t x+(1-t) y) \leq t f(x)+(1-t) f(y),
$$

for all $x, y \in I$ and $t \in[0,1]$.

Several generalizations of convex functions including quasiconvex, $s$-convex, $m$ convex, MT-convex, $h$-convex, $\eta$-convex and so on has been provided over the years. Of particular interest is the generalization of convexity to $(\alpha, m)$-convexity by Miheşan [20] as follows.

Definition 1.1. A function $f:[0, d] \subset \mathbb{R} \rightarrow \mathbb{R}, d>0$ is said to be $(\alpha, m)$-convex function where $(\alpha, m) \in[0,1]^{2}$ if

$$
f(t x+m(1-t) y) \leq t^{\alpha} f(x)+m\left(1-t^{\alpha}\right) f(y),
$$

for all $x, y \in[0, d]$ and $t \in[0,1]$.

Remark 1.2. If we choose $(\alpha, m)=(1, m)$, then we obtain $m$-convex functions and if $(\alpha, m)=(1,1)$, then we have the ordinary convex functions. 
For some recent generalizations and results related to $(\alpha, m)$-convex functions, we refer the interested reader to the papers $[6,23,24,28,29,25,14,16]$.

In [26], Polyak gave the following extension of convex functions known as strongly convex functions.

Definition 1.3. Given an interval $I$ in $\mathbb{R}$, a function $f: I \rightarrow \mathbb{R}$ is said to be strongly convex with modulus $\mu \geq 0$ if

$$
f(t x+(1-t) y) \leq t f(x)+(1-t) f(y)-\mu t(1-t)(y-x)^{2},
$$

for all $x, y \in I$ and $t \in[0,1]$.

The introduction of the notion of strong convexity has led many authors to extend the other classes of convex functions in a similar way (see $[17,7,14,5,4,15]$ ). In a similar way, we have the following definition of strongly $(\alpha, m)$-convex functions.

Definition 1.4. A function $f:[0, d] \subset \mathbb{R} \rightarrow \mathbb{R}, d>0$ is said to be strongly $(\alpha, m)$ convex with modulus $\mu \geq 0$ for $(\alpha, m) \in[0,1]^{2}$ if

$$
f(t x+m(1-t) y) \leq t^{\alpha} f(x)+m\left(1-t^{\alpha}\right) f(y)-\mu t(1-t)(y-x)^{2},
$$

for all $x, y \in[0, d]$ and $t \in[0,1]$.

Remark 1.5. If we choose $(\alpha, m)=(1, m)$ in Definition 1.4 , then we obtain strongly $m$-convex functions and if $(\alpha, m)=(1,1)$, then we have the strongly convex functions.

In 1938, Ostrowski [22] obtained the following inequality which is known in the literature as Ostrowski inequality.

Theorem 1.6. Let $f:[a, b] \rightarrow \mathbb{R}$ be continuous on $[a, b]$ and differentiable in $(a, b)$ and its derivative $f^{\prime}:(a, b) \rightarrow \mathbb{R}$ is bounded in $(a, b)$. If $M:=\sup _{t \in(a, b)}\left|f^{\prime}(t)\right|<\infty$, then we have

$$
\left|f(x)-\frac{1}{b-a} \int_{a}^{b} f(t) d t\right| \leq\left(\frac{1}{4}+\frac{\left(x-\frac{a+b}{2}\right)^{2}}{(b-a)^{2}}\right)(b-a) M,
$$

for all $x \in[a, b]$. The inequality is sharp in the sense that the constant $\frac{1}{4}$ cannot be replaced by a smaller one.

Many authors have studied and generalized this inequality in several different ways. For more information about the Ostrowski inequality and its associates, we refer the reader to the papers $[1,2,3,8,9,10,11,12,13,18,19,27,25]$. The authors in $[13,18,19,27,1,8,25]$ provided some Ostrowski type inequalities for some classes of convex functions.

Motivated by the above results, the main goal of this paper is to provide some Ostrowski type integral inequalities for functions whose derivatives at some powers are strongly $(\alpha, m)$-convex via the $k$-Riemman-Liouville fractional integrals. We complete this section with the defiinition of the $k$-Riemann-Liouville fractional integrals.

Definition 1.7 (See [21]). The Riemann-Liouville $k$-fractional integrals of order $\beta>0$, for a real-valued continuous function $f$ are defined as

$$
{ }_{k} J_{a^{+}}^{\beta} f(x)=\frac{1}{k \Gamma_{k}(\beta)} \int_{a}^{x}(x-t)^{\frac{\beta}{k}-1} f(t) d t, x>a,
$$


and

$$
{ }_{k} J_{b^{-}}^{\beta} f(x)=\frac{1}{k \Gamma_{k}(\beta)} \int_{x}^{b}(t-x)^{\frac{\beta}{k}-1} f(t) d t, x<b,
$$

where $k>0$, and $\Gamma_{k}$ is the $k$-gamma function given by

$$
\Gamma_{k}(x)=\int_{0}^{\infty} t^{x-1} e^{-\frac{t^{k}}{k}} d t, \operatorname{Re}(x)>0
$$

with the properties that $\Gamma_{k}(x+k)=x \Gamma_{k}(x)$ and $\Gamma_{k}(k)=1$.

Remark 1.8. If $k=1$ in Definition 1.7, then we have the classical Riemann-Liouville fractional integrals and if $\beta=k=1$, then we obtain the classical Riemann integral.

\section{Main results}

To prove our results we need the following result which is given by Farid and Usman [13].

Lemma 2.1 ([13]). Let $f:[a, b] \rightarrow \mathbb{R}$ be a differentiable function on $(a, b)$ with $a<b$ such that $f^{\prime} \in L_{1}([a, b])$, then for all $x \in[a, b]$ and $\beta, k>0$, the following equality holds;

$$
\begin{gathered}
\frac{(x-a)^{\frac{\beta}{k}}+(b-x)^{\frac{\beta}{k}}}{b-a} f(x)-\frac{\Gamma_{k}(\beta+k)}{b-a}\left[{ }_{k} J_{x^{-}}^{\beta} f(a)+{ }_{k} J_{x^{+}}^{\beta} f(b)\right] \\
=\frac{(x-a)^{\frac{\beta}{k}+1}}{b-a} \int_{0}^{1} t^{\frac{\beta}{k}} f^{\prime}(t x+(1-t) a) d t-\frac{(b-x)^{\frac{\beta}{k}+1}}{b-a} \int_{0}^{1} t^{\frac{\beta}{k}} f^{\prime}(t x+(1-t) b) d t .
\end{gathered}
$$

Theorem 2.2. Let $f:[0, \infty) \rightarrow \mathbb{R}$ be a differentiable function on $(0, \infty)$ such that $f^{\prime} \in L_{1}([a, b])$ with $0 \leq a<b$. If $\left|f^{\prime}\right|$ is strongly $(\alpha, m)$-convex with modulus $\mu \geq 0$ for $\alpha \in[0,1]$ and $m \in(0,1]$, then the inequality

$$
\begin{gathered}
\left|\frac{(x-a)^{\frac{\beta}{k}}+(b-x)^{\frac{\beta}{k}}}{b-a} f(x)-\frac{\Gamma_{k}(\beta+k)}{b-a}\left[{ }_{k} J_{x^{-}}^{\beta} f(a)+{ }_{k} J_{x^{+}}^{\beta} f(b)\right]\right| \\
\leq \frac{(x-a)^{\frac{\beta}{k}+1}}{b-a}\left[\frac{\left|f^{\prime}(x)\right|}{\frac{\beta}{k}+\alpha+1}+\frac{\alpha m\left|f^{\prime}\left(\frac{a}{m}\right)\right|}{\left(\frac{\beta}{k}+1\right)\left(\frac{\beta}{k}+\alpha+1\right)}\right. \\
\left.-\frac{\mu\left(x-\frac{a}{m}\right)^{2}}{\left(\frac{\beta}{k}+2\right)\left(\frac{\beta}{k}+3\right)}\right] \\
+\frac{(b-x)^{\frac{\beta}{k}+1}}{b-a}\left[\frac{\left|f^{\prime}(x)\right|}{\frac{\beta}{k}+\alpha+1}+\frac{\alpha m\left|f^{\prime}\left(\frac{b}{m}\right)\right|}{\left(\frac{\beta}{k}+1\right)\left(\frac{\beta}{k}+\alpha+1\right)}\right. \\
\left.-\frac{\mu\left(\frac{b}{m}-x\right)^{2}}{\left(\frac{\beta}{k}+2\right)\left(\frac{\beta}{k}+3\right)}\right]
\end{gathered}
$$

holds for all $x \in[a, b]$ and $\beta, k>0$. 
Proof. Using Lemma 2.1 and the strong $(\alpha, m)$-convexity of $\left|f^{\prime}\right|$, we have that

$$
\begin{aligned}
& \left|\frac{(x-a)^{\frac{\beta}{k}}+(b-x)^{\frac{\beta}{k}}}{b-a} f(x)-\frac{\Gamma_{k}(\beta+k)}{b-a}\left[{ }_{k} J_{x^{-}}^{\beta} f(a)+{ }_{k} J_{x^{+}}^{\beta} f(b)\right]\right| \\
& \leq \frac{(x-a)^{\frac{\beta}{k}+1}}{b-a} \int_{0}^{1} t^{\frac{\beta}{k}}\left|f^{\prime}(t x+(1-t) a)\right| d t \\
& +\frac{(b-x)^{\frac{\beta}{k}+1}}{b-a} \int_{0}^{1} t^{\frac{\beta}{k}}\left|f^{\prime}(t x+(1-t) b)\right| d t \\
& \leq \frac{(x-a)^{\frac{\beta}{k}+1}}{b-a} \int_{0}^{1} t^{\frac{\beta}{k}}\left(t^{\alpha}\left|f^{\prime}(x)\right|+m\left(1-t^{\alpha}\right)\left|f^{\prime}\left(\frac{a}{m}\right)\right|-\mu t(1-t)\left(x-\frac{a}{m}\right)^{2}\right) d t \\
& +\frac{(b-x)^{\frac{\beta}{k}+1}}{b-a} \int_{0}^{1} t^{\frac{\beta}{k}}\left(t^{\alpha}\left|f^{\prime}(x)\right|+m\left(1-t^{\alpha}\right)\left|f^{\prime}\left(\frac{b}{m}\right)\right|-\mu t(1-t)\left(\frac{b}{m}-x\right)^{2}\right) d t .
\end{aligned}
$$

That is,

$$
\begin{gathered}
\left|\frac{(x-a)^{\frac{\beta}{k}}+(b-x)^{\frac{\beta}{k}}}{b-a} f(x)-\frac{\Gamma_{k}(\beta+k)}{b-a}\left[k J_{x^{-}}^{\beta} f(a)+{ }_{k} J_{x^{+}}^{\beta} f(a)\right]\right| \\
\leq \frac{(x-a)^{\frac{\beta}{k}+1}}{b-a}\left[\left|f^{\prime}(x)\right| \int_{0}^{1} t^{\frac{\beta}{k}+\alpha} d t+m\left|f^{\prime}\left(\frac{a}{m}\right)\right| \int_{0}^{1} t^{\frac{\beta}{k}}\left(1-t^{\alpha}\right) d t\right. \\
\left.\quad-\mu\left(x-\frac{a}{m}\right)^{2} \int_{0}^{1} t^{\frac{\beta}{k}+1}(1-t) d t\right] \\
+\frac{(b-x)^{\frac{\beta}{k}+1}}{b-a}\left[\left|f^{\prime}(x)\right| \int_{0}^{1} t^{\frac{\beta}{k}+\alpha} d t+m\left|f^{\prime}\left(\frac{b}{m}\right)\right| \int_{0}^{1} t^{\frac{\beta}{k}}\left(1-t^{\alpha}\right) d t\right. \\
\left.-\mu\left(\frac{b}{m}-x\right)^{2} \int_{0}^{1} t^{\frac{\beta}{k}+1}(1-t) d t\right] .
\end{gathered}
$$

The desired inequality follows from (2.1) by using the fact that

$$
\begin{gathered}
\int_{0}^{1} t^{\frac{\beta}{k}+\alpha} d t=\frac{1}{\frac{\beta}{k}+\alpha+1} \\
\int_{0}^{1} t^{\frac{\beta}{k}}\left(1-t^{\alpha}\right) d t=\frac{\alpha}{\left(\frac{\beta}{k}+1\right)\left(\frac{\beta}{k}+\alpha+1\right)}
\end{gathered}
$$

and

$$
\int_{0}^{1} t^{\frac{\beta}{k}+1}(1-t) d t=\frac{1}{\left(\frac{\beta}{k}+2\right)\left(\frac{\beta}{k}+3\right)}
$$

Corollary 2.3. Let $f:[0, \infty) \rightarrow \mathbb{R}$ be a differentiable function on $(0, \infty)$ such that $f^{\prime} \in L_{1}([a, b])$ with $0 \leq a<b$. If $\left|f^{\prime}\right|$ is $(\alpha, m)$-convex for $\alpha \in[0,1]$ and $m \in(0,1]$, 
then the inequality

$$
\begin{aligned}
& \left|\frac{(x-a)^{\frac{\beta}{k}}+(b-x)^{\frac{\beta}{k}}}{b-a} f(x)-\frac{\Gamma_{k}(\beta+k)}{b-a}\left[{ }_{k} J_{x^{-}}^{\beta} f(a)+{ }_{k} J_{x^{+}}^{\beta} f(b)\right]\right| \\
& \leq \frac{(x-a)^{\frac{\beta}{k}+1}}{b-a}\left[\frac{\left|f^{\prime}(x)\right|}{\frac{\beta}{k}+\alpha+1}+\frac{\alpha m\left|f^{\prime}\left(\frac{a}{m}\right)\right|}{\left(\frac{\beta}{k}+1\right)\left(\frac{\beta}{k}+\alpha+1\right)}\right] \\
& +\frac{(b-x)^{\frac{\beta}{k}+1}}{b-a}\left[\frac{\left|f^{\prime}(x)\right|}{\frac{\beta}{k}+\alpha+1}+\frac{\alpha m\left|f^{\prime}\left(\frac{b}{m}\right)\right|}{\left(\frac{\beta}{k}+1\right)\left(\frac{\beta}{k}+\alpha+1\right)}\right]
\end{aligned}
$$

holds for all $x \in[a, b]$ and $\beta, k>0$.

Proof. Take $\mu=0$ in Theorem 2.2.

Theorem 2.4. Let $f:[0, \infty) \rightarrow \mathbb{R}$ be a differentiable function on $(0, \infty)$ such that $f^{\prime} \in L_{1}([a, b])$ with $0 \leq a<b$. If $\left|f^{\prime}\right|^{q}$ is strongly $(\alpha, m)$-convex with modulus $\mu \geq 0$ for $q>1, \alpha \in[0,1]$ and $m \in(0,1]$, then the inequality

$$
\begin{aligned}
& \left|\frac{(x-a)^{\frac{\beta}{k}}+(b-x)^{\frac{\beta}{k}}}{b-a} f(x)-\frac{\Gamma_{k}(\beta+k)}{b-a}\left[{ }_{k} J_{x^{-}}^{\beta} f(a)+{ }_{k} J_{x^{+}}^{\beta} f(b)\right]\right| \\
& \leq \frac{(x-a)^{\frac{\beta}{k}+1}}{(b-a)\left(\frac{\beta}{k} p+1\right)^{\frac{1}{p}}}\left(\frac{\left|f^{\prime}(x)\right|^{q}}{\alpha+1}+\frac{\alpha m\left|f^{\prime}\left(\frac{a}{m}\right)\right|^{q}}{\alpha+1}-\frac{\mu\left(x-\frac{a}{m}\right)^{2}}{6}\right)^{\frac{1}{q}} \\
& \quad+\frac{(b-x)^{\frac{\beta}{k}+1}}{(b-a)\left(\frac{\beta}{k} p+1\right)^{\frac{1}{p}}}\left(\frac{\left|f^{\prime}(x)\right|^{q}}{\alpha+1}+\frac{\alpha m\left|f^{\prime}\left(\frac{b}{m}\right)\right|^{q}}{\alpha+1}-\frac{\mu\left(\frac{b}{m}-x\right)^{2}}{6}\right)^{\frac{1}{q}}
\end{aligned}
$$

holds for all $x \in[a, b]$ and $\beta, k>0$, for $\frac{1}{p}+\frac{1}{q}=1$.

Proof. Using Lemma 2.1, the Hölder's inequality and the strong $(\alpha, m)$-convexity of $\left|f^{\prime}\right|^{q}$, we have that

$$
\begin{gathered}
\left|\frac{(x-a)^{\frac{\beta}{k}}+(b-x)^{\frac{\beta}{k}}}{b-a} f(x)-\frac{\Gamma_{k}(\beta+k)}{b-a}\left[k J_{x^{-}}^{\beta} f(a)+{ }_{k} J_{x^{+}}^{\beta} f(b)\right]\right| \\
\leq \frac{(x-a)^{\frac{\beta}{k}+1}}{b-a} \int_{0}^{1} t^{\frac{\beta}{k}}\left|f^{\prime}(t x+(1-t) a)\right| d t \\
+\frac{(b-x)^{\frac{\beta}{k}+1}}{b-a} \int_{0}^{1} t^{\frac{\beta}{k}}\left|f^{\prime}(t x+(1-t) b)\right| d t \\
\leq \frac{(x-a)^{\frac{\beta}{k}+1}}{b-a}\left(\int_{0}^{1} t^{\frac{\beta}{k} p} d t\right)^{\frac{1}{p}}\left(\int_{0}^{1}\left|f^{\prime}(t x+(1-t) a)\right|^{q} d t\right)^{\frac{1}{q}} \\
+\frac{(b-x)^{\frac{\beta}{k}+1}}{b-a}\left(\int_{0}^{1} t^{\frac{\beta}{k} p} d t\right)^{\frac{1}{p}}\left(\int_{0}^{1}\left|f^{\prime}(t x+(1-t) b)\right|^{q} d t\right)^{\frac{1}{q}} \\
\leq \frac{(x-a)^{\frac{\beta}{k}+1}}{b-a}\left(\int_{0}^{1} t^{\frac{\beta}{k} p} d t\right)^{\frac{1}{p}}\left(\int _ { 0 } ^ { 1 } \left(t^{\alpha}\left|f^{\prime}(x)\right|^{q}+m\left(1-t^{\alpha}\right)\left|f^{\prime}\left(\frac{a}{m}\right)\right|^{q}\right.\right.
\end{gathered}
$$




$$
\begin{gathered}
\left.\left.-\mu t(1-t)\left(x-\frac{a}{m}\right)^{2}\right) d t\right)^{\frac{1}{q}} \\
+\frac{(b-x)^{\frac{\beta}{k}+1}}{b-a}\left(\int_{0}^{1} t^{\frac{\beta}{k} p} d t\right)^{\frac{1}{p}}\left(\int _ { 0 } ^ { 1 } \left(t^{\alpha}\left|f^{\prime}(x)\right|^{q}+m\left(1-t^{\alpha}\right)\left|f^{\prime}\left(\frac{b}{m}\right)\right|^{q}\right.\right. \\
\left.\left.-\mu t(1-t)\left(\frac{b}{m}-x\right)^{2}\right) d t\right)^{\frac{1}{q}} \\
\leq \frac{(x-a)^{\frac{\beta}{k}+1}}{(b-a)\left(\frac{\beta}{k} p+1\right)^{\frac{1}{p}}}\left(\frac{\left|f^{\prime}(x)\right|^{q}}{\alpha+1}+\frac{\alpha m\left|f^{\prime}\left(\frac{a}{m}\right)\right|^{q}}{\alpha+1}-\frac{\mu\left(x-\frac{a}{m}\right)^{2}}{6}\right)^{\frac{1}{q}} \\
+\frac{(b-x)^{\frac{\beta}{k}+1}}{(b-a)\left(\frac{\beta}{k} p+1\right)^{\frac{1}{p}}}\left(\frac{\left|f^{\prime}(x)\right|^{q}}{\alpha+1}+\frac{\alpha m\left|f^{\prime}\left(\frac{b}{m}\right)\right|^{q}}{\alpha+1}-\frac{\mu\left(\frac{b}{m}-x\right)^{2}}{6}\right)^{\frac{1}{q}}
\end{gathered}
$$

This completes the proof.

Corollary 2.5. Let $f:[0, \infty) \rightarrow \mathbb{R}$ be a differentiable function on $(0, \infty)$ such that $f^{\prime} \in L_{1}([a, b])$ with $0 \leq a<b$. If $\left|f^{\prime}\right|^{q}$ is $(\alpha, m)$-convex for $q>1, \alpha \in[0,1]$ and $m \in(0,1]$, then the inequality

$$
\begin{gathered}
\left|\frac{(x-a)^{\frac{\beta}{k}}+(b-x)^{\frac{\beta}{k}}}{b-a} f(x)-\frac{\Gamma_{k}(\beta+k)}{b-a}\left[{ }_{k} J_{x^{-}}^{\beta} f(a)+{ }_{k} J_{x^{+}}^{\beta} f(b)\right]\right| \\
\leq \frac{(x-a)^{\frac{\beta}{k}+1}}{(b-a)\left(\frac{\beta}{k} p+1\right)^{\frac{1}{p}}}\left(\frac{\left|f^{\prime}(x)\right|^{q}}{\alpha+1}+\frac{\alpha m\left|f^{\prime}\left(\frac{a}{m}\right)\right|^{q}}{\alpha+1}\right)^{\frac{1}{q}} \\
\quad+\frac{(b-x)^{\frac{\beta}{k}}+1}{(b-a)\left(\frac{\beta}{k} p+1\right)^{\frac{1}{p}}}\left(\frac{\left|f^{\prime}(x)\right|^{q}}{\alpha+1}+\frac{\alpha m\left|f^{\prime}\left(\frac{b}{m}\right)\right|^{q}}{\alpha+1}\right)^{\frac{1}{q}}
\end{gathered}
$$

holds for all $x \in[a, b]$ and $\beta, k>0$, for $\frac{1}{p}+\frac{1}{q}=1$.

Proof. Take $\mu=0$ in Theorem 2.4.

Theorem 2.6. Let $f:[0, \infty) \rightarrow \mathbb{R}$ be a differentiable function on $(0, \infty)$ such that $f^{\prime} \in L_{1}([a, b])$ with $0 \leq a<b$. If $\left|f^{\prime}\right|^{q}$ is strongly $(\alpha, m)$-convex with modulus $\mu \geq 0$ for $q>1, \alpha \in[0,1]$ and $m \in(0,1]$, then the inequality

$$
\begin{gathered}
\left|\frac{(x-a)^{\frac{\beta}{k}}+(b-x)^{\frac{\beta}{k}}}{b-a} f(x)-\frac{\Gamma_{k}(\beta+k)}{b-a}\left[{ }_{k} J_{x^{-}}^{\beta} f(a)+{ }_{k} J_{x^{+}}^{\beta} f(b)\right]\right| \\
\leq \frac{(x-a)^{\frac{\beta}{k}+1}}{(b-a)\left(\frac{\beta}{k}+1\right)^{\frac{1}{p}}}\left(\frac{\left|f^{\prime}(x)\right|^{q}}{\frac{\beta}{k}+\alpha+1}+\frac{\alpha m\left|f^{\prime}\left(\frac{a}{m}\right)\right|^{q}}{\left(\frac{\beta}{k}+1\right)\left(\frac{\beta}{k}+\alpha+1\right)}-\frac{\mu\left(x-\frac{a}{m}\right)^{2}}{\left(\frac{\beta}{k}+2\right)\left(\frac{\beta}{k}+3\right)}\right)^{\frac{1}{q}} \\
+\frac{(b-x)^{\frac{\beta}{k}+1}}{(b-a)\left(\frac{\beta}{k}+1\right)^{\frac{1}{p}}}\left(\frac{\left|f^{\prime}(x)\right|^{q}}{\frac{\beta}{k}+\alpha+1}+\frac{\alpha m\left|f^{\prime}\left(\frac{b}{m}\right)\right|^{q}}{\left(\frac{\beta}{k}+1\right)\left(\frac{\beta}{k}+\alpha+1\right)}-\frac{\mu\left(\frac{b}{m}-x\right)^{2}}{\left(\frac{\beta}{k}+2\right)\left(\frac{\beta}{k}+3\right)}\right)^{\frac{1}{q}}
\end{gathered}
$$

holds for all $x \in[a, b]$ and $\beta, k>0$, for $\frac{1}{p}+\frac{1}{q}=1$. 
Proof. Using Lemma 2.1, the Hölder's inequality and the strong $(\alpha, m)$-convexity of $\left|f^{\prime}\right|^{q}$, we have that

$$
\begin{aligned}
& \left|\frac{(x-a)^{\frac{\beta}{k}}+(b-x)^{\frac{\beta}{k}}}{b-a} f(x)-\frac{\Gamma_{k}(\beta+k)}{b-a}\left[k J_{x^{-}}^{\beta} f(a)+{ }_{k} J_{x^{+}}^{\beta} f(b)\right]\right| \\
& \leq \frac{(x-a)^{\frac{\beta}{k}+1}}{b-a} \int_{0}^{1} t^{\frac{\beta}{k}}\left|f^{\prime}(t x+(1-t) a)\right| d t \\
& +\frac{(b-x)^{\frac{\beta}{k}+1}}{b-a} \int_{0}^{1} t^{\frac{\beta}{k}}\left|f^{\prime}(t x+(1-t) b)\right| d t \\
& \leq \frac{(x-a)^{\frac{\beta}{k}+1}}{b-a}\left(\int_{0}^{1} t^{\frac{\beta}{k}} d t\right)^{\frac{1}{p}}\left(\int_{0}^{1} t^{\frac{\beta}{k}}\left|f^{\prime}(t x+(1-t) a)\right|^{q} d t\right)^{\frac{1}{q}} \\
& +\frac{(b-x)^{\frac{\beta}{k}+1}}{b-a}\left(\int_{0}^{1} t^{\frac{\beta}{k}} d t\right)^{\frac{1}{p}}\left(\int_{0}^{1} t^{\frac{\beta}{k}}\left|f^{\prime}(t x+(1-t) b)\right|^{q} d t\right)^{\frac{1}{q}} \\
& \leq \frac{(x-a)^{\frac{\beta}{k}+1}}{b-a}\left(\int_{0}^{1} t^{\frac{\beta}{k}} d t\right)^{\frac{1}{p}}\left(\int _ { 0 } ^ { 1 } t ^ { \frac { \beta } { k } } \left(t^{\alpha}\left|f^{\prime}(x)\right|^{q}+m\left(1-t^{\alpha}\right)\left|f^{\prime}\left(\frac{a}{m}\right)\right|^{q}\right.\right. \\
& \left.\left.-\mu t(1-t)\left(x-\frac{a}{m}\right)^{2}\right) d t\right)^{\frac{1}{q}} \\
& +\frac{(b-x)^{\frac{\beta}{k}+1}}{b-a}\left(\int_{0}^{1} t^{\frac{\beta}{k}} d t\right)^{\frac{1}{p}}\left(\int _ { 0 } ^ { 1 } t ^ { \frac { \beta } { k } } \left(t^{\alpha}\left|f^{\prime}(x)\right|^{q}+m\left(1-t^{\alpha}\right)\left|f^{\prime}\left(\frac{b}{m}\right)\right|^{q}\right.\right. \\
& \left.\left.-\mu t(1-t)\left(\frac{b}{m}-x\right)^{2}\right) d t\right)^{\frac{1}{q}} \\
& \leq \frac{(x-a)^{\frac{\beta}{k}+1}}{(b-a)\left(\frac{\beta}{k}+1\right)^{\frac{1}{p}}}\left(\left|f^{\prime}(x)\right|^{q} \int_{0}^{1} t^{\frac{\beta}{k}+\alpha} d t+m\left|f^{\prime}\left(\frac{a}{m}\right)\right|^{q} \int_{0}^{1} t^{\frac{\beta}{k}}\left(1-t^{\alpha}\right) d t\right. \\
& \left.-\mu\left(x-\frac{a}{m}\right)^{2} \int_{0}^{1} t^{\frac{\beta}{k}+1}(1-t) d t\right)^{\frac{1}{q}} \\
& +\frac{(b-x)^{\frac{\beta}{k}+1}}{(b-a)\left(\frac{\beta}{k}+1\right)^{\frac{1}{p}}}\left(\left|f^{\prime}(x)\right|^{q} \int_{0}^{1} t^{\frac{\beta}{k}+\alpha} d t+m\left|f^{\prime}\left(\frac{b}{m}\right)\right|^{q} \int_{0}^{1} t^{\frac{\beta}{k}}\left(1-t^{\alpha}\right) d t\right. \\
& \left.-\mu\left(\frac{b}{m}-x\right)^{2} \int_{0}^{1} t^{\frac{\beta}{k}+1}(1-t) d t\right)^{\frac{1}{q}} .
\end{aligned}
$$

By using (2.2), (2.3), (2.4) and (2.5), we have the desired inequality.

Corollary 2.7. Let $f:[0, \infty) \rightarrow \mathbb{R}$ be a differentiable function on $(0, \infty)$ such that $f^{\prime} \in L_{1}([a, b])$ with $0 \leq a<b$. If $\left|f^{\prime}\right|^{q}$ is $(\alpha, m)$-convex for $q>1, \alpha \in[0,1]$ and $m \in(0,1]$, then the inequality

$$
\left|\frac{(x-a)^{\frac{\beta}{k}}+(b-x)^{\frac{\beta}{k}}}{b-a} f(x)-\frac{\Gamma_{k}(\beta+k)}{b-a}\left[{ }_{k} J_{x^{-}}^{\beta} f(a)+{ }_{k} J_{x^{+}}^{\beta} f(b)\right]\right|
$$




$$
\begin{aligned}
& \leq \frac{(x-a)^{\frac{\beta}{k}+1}}{(b-a)\left(\frac{\beta}{k}+1\right)^{\frac{1}{p}}}\left(\frac{\left|f^{\prime}(x)\right|^{q}}{\frac{\beta}{k}+\alpha+1}+\frac{\alpha m\left|f^{\prime}\left(\frac{a}{m}\right)\right|^{q}}{\left(\frac{\beta}{k}+1\right)\left(\frac{\beta}{k}+\alpha+1\right)}\right)^{\frac{1}{q}} \\
& +\frac{(b-x)^{\frac{\beta}{k}+1}}{(b-a)\left(\frac{\beta}{k}+1\right)^{\frac{1}{p}}}\left(\frac{\left|f^{\prime}(x)\right|^{q}}{\frac{\beta}{k}+\alpha+1}+\frac{\alpha m\left|f^{\prime}\left(\frac{b}{m}\right)\right|^{q}}{\left(\frac{\beta}{k}+1\right)\left(\frac{\beta}{k}+\alpha+1\right)}\right)^{\frac{1}{q}}
\end{aligned}
$$

holds for all $x \in[a, b]$ and $\beta, k>0$, for $\frac{1}{p}+\frac{1}{q}=1$.

Proof. Take $\mu=0$ in Theorem 2.6.

\section{Conclusion}

New Ostrowski type inequalities for functions whose derivatives in absolute value at certain powers are strongly $(\alpha, m)$-convex and $(\alpha, m)$-convex functions via the $k$ Riemman-Liouville fractional integrals has been provided. Similar results could be obtained for $m$ and strongly $m$-convex functions as particular cases. Also several other interesting inequalities could be obtained by considering different values of the parameters $\beta$ and $k$. For instance, if $k=1$, then the results will be in terms of the classical Riemann-Liouville fractional integrals and if $\beta=k=1$, then we have the results with the integrals in the classical Riemann sense. The details are left for the interested reader.

Acknowledgement. The author wishes to express his profound gratitude to the anonymous referee for his/her careful reading of the manuscript and the very useful comments that have been implemented in the final version of the manuscript.

\section{References}

[1] Alomari, M., Darus, M., Dragomir, S.S., Cerone, P., Ostrowski type inequalities for the functions whose derivative are s-convex in second sense, Appl. Math. Lett., 23(2010), no. 9, 1071-1076.

[2] Anastassiou, G.A., Ostrowski type inequalities, Proc. Amer. Math. Soc., 123 (1995), no. $12,3775-3781$.

[3] Anastassiou, G.A., Hooshmandasl, M.R., Ghasemi, A., Moftakharzadeh, F., Montgomery identities for fractional integrals and related inequalities, J. Inequal. Pure and Appl. Math., 10(2009), no. 4, Art. 97, 6 pp.

[4] Angulo, H., Giménez, J., Moros, A., Nikodem, K., On strongly h-convex functions, Ann. Funct. Anal., 2(2011), no. 2, 85-91.

[5] Awan, M.U., Noorb, M.A., Noorb, K.I., Safdar, F., On strongly generalized convex functions, FILOMAT, 31(2017), no. 18, 5783-5790.

[6] Bakula, M.K., Özdemir, M.E., Pečarić, J., Hadamard-type inequalities for $m$-convex and $(\alpha, m)$-convex functions, J. Inequal. Pure and Appl. Math., 9(2007), no. 4, Art. 96.

[7] Bracamonte, M., Giménez, J., Vivas-Cortez, M., Hermite-Hadamard-Fejér type inequalities for strongly $(s, m)$-convex functions with modulus $c$, in second sense, Appl. Math. Inf. Sci., 10(2016), no. 6, 2045-2053. 
[8] Dragomir, S.S., An Ostrowski type inequality for convex functions, Univ. Beograd. Publ. Elektrotehn. Fak. Ser. Mat., 16(2005), 12-25.

[9] Dragomir, S.S., A generalization of Ostrowsk integral inequality for mappings whose derivatives belong to $L_{1}[a, b]$ and applications in numerical integration, J. Comput. Anal. Appl., 3(2001), no. 4, 343-360.

[10] Dragomir, S.S., A generalization of the Ostrowski integral inequality for mappings whose derivatives belong to $L_{p}[a, b]$ and applications in numerical integration, J. Math. Anal. Appl., 255(2001), 605-626.

[11] Dragomir, S.S., Wang, S., A new inequality of Ostrowski's type in $L_{1}$-norm and applications to some special means and to some numerical quadrature rules, Tamkang J. Math., 28(1997), 239-244.

[12] Dragomir, S.S., Wang, S., A new inequality of Ostrowski's type in $L_{p}$-norm, Indian J. Math., 40(1998), no. 3, 299-304.

[13] Farid, G., Usman, M., Ostrowski type $k$-fractional integral inequalities for MT-convex and h-convex functions, Nonlinear Funct. Anal. Appl., 22(2017), no. 3, 627-639.

[14] He, C.Y., Wang, Y., Xi, B.Y., Qi, F., Hermite-Hadamard type inequalities for $(\alpha, m)-H A$ and strongly $(\alpha, m)-H A$ convex functions, J. Nonlinear Sci. Appl., 10(2017), 205-214.

[15] Hua, J., Xi, B.Y., Qi, F., Some new inequalities of Simpson type for strongly s-convex function, Afr. Mat., 26(2015), 741-752.

[16] Iscan, I., Kadakal, H., Kadakal, M., Some new integral inequalities for functions whose $n$th derivatives in absolute value are $(\alpha, m)$-convex functions, New Trends Math. Sci., 5(2017), no. 2, 180-185.

[17] Lara, T., Merentes, N., Quintero, R., Rosales, E., On strongly m-convex functions, Math. Aeterna, 5(2015), no. 3, 521-535.

[18] Liu, W., Ostrowski type fractional integral inequalities for MT-convex function, Miskolc Math. Notes, 16(2015), no. 1, 249-256.

[19] Liu, W., Some Ostrowski type inequalities via Riemann-Liouville fractional integral for h-convex function, J. Comput. Anal. Appl., 16(2014), no. 1, 998-1004.

[20] Miheşan, V.G., A generalization of the convexity, Seminar on Functional Equations, Approximation and Convexity, Cluj-Napoca, Romania, 1993.

[21] Mubeen, S., Habibullah, G.M., k-Fractional integrals and applications, Int. J. Contemp. Math. Sci., 7(2012), no. 2, 89-94.

[22] Ostrowski, A.M., Über die Absolutabweichung einer differentiebaren Funktion von ihrem Integralmitelwert, Comment. Math. Helv., 10(1938), 226-227.

[23] Özdemir, M.E., Akdemir, A.O., Ekinci, A., New Hadamard-type inequalities for functions whose derivatives are $(\alpha, m)$-convex functions, Tbilisi Math. J., 7(2014), no. 2, 61-72.

[24] Özdemir, M.E., Avci, M., Kavurmaci, H., Hermite-Hadamard-type inequalities via ( $\alpha, m)$-convexity, Comput. Math. Appl., 61(2011), 2614-2620.

[25] Özdemir, M.E., Kavurmaci, H., Set, E., Ostrowski type inequalities for $(\alpha, m)$-convex functions, Kyungpook Math. J., 50(2010), 371-378.

[26] Polyak, B.T., Existence theorems and convergence of minimizing sequences in extremum problems with restrictions, Soviet Math. Dokl., 7(1966), 72-75.

[27] Set, E., New inequalities of Ostrowski type for mappings whose derivatives are s-convex in the second sense via fractional integrals, Comput. Math. Appl., 63(2012), 1147-1154. 
[28] Set, E., Sardari, M., Özdemir, M.E., Rooin, J., On generalizations of the Hadamard inequality for $(\alpha, m)$-convex functions, RGMIA Res. Rep. Coll., 12(2009), no. 4, Art. 4.

[29] Sun, W., Liu, Q., New Hermite-Hadamard type inequalities for $(\alpha, m)$-convex functions and applications to special means, J. Math. Inequal., 11(2017), no. 2, 383-397.

Seth Kermausuor

Alabama State University

Department of Mathematics and Computer Science

Montgomery, AL 36101, U.S.A.

e-mail: skermausour@alasu.edu 